\title{
Die Anfänge des tschechoslowakischen Parlamentarismus 1918-1920*
}

The article deals with the constitutional development of Czechoslovakia from its creation (28th October 1918) to the adoption of its Constitutional Charter (29th February 1920). It looks for similarities and differences between the constitutional laws and parliamentary rules of procedure of the Habsburg Monarchy and the new republic. Also, it observes the discussions in the Constitutional Committee and in the plenary sessions of the National Assembly and compares the Rules of Procedure of the House of Commons of the Reichsrat of 1875 and Act No. 325/1920, On the Rules of Procedure of the House of Commons of the National Assembly. Despite the change of state form there were, especially at the level of parliamentary rules, many similarities which resulted from a common tradition and the experiences of leading Czech politicians in the Viennese parliament.

\section{Einleitung}

In den Jahren 1918-1920 entstanden die politische Ordnung und das Verfassungssystem der Tschechoslowakei, das seinen demokratischen Charakter bis zum Ende der Ersten Republik (Herbst 1938) behielt. Dieses System zeigt interessante spezifische Merkmale, vor allem (neben der starken inoffiziellen Rolle des Präsidenten T. G. Masaryk) die immense Bedeutung der Parteienkoalitionen und den Grundsatz, dass Parlamentsmandate in der Verfügungsgewalt der Parteien und nicht der einzelnen Abgeordneten standen. Für die tschechoslowakische Verfassung vom 29. Februar 1920 waren amerikanische und französische Vorbilder maßgeblich. Sie wurde jedoch auch von der langjährigen politischen (und auch verfassungs- sowie verwaltungsrechtlichen) Erfahrung der führenden tschechischen Politiker in der Habsburgermonarchie beeinflusst. Der vorliegende Beitrag stellt die Entstehung des parlamentarischen Systems der Tschechoslowakei anhand der parlamentarischen Materialien vor und arbeitet
Ähnlichkeiten und Unterschiede im Vergleich mit der Habsburgermonarchie heraus.

\section{Die provisorische Verfassung}

Der Erste Weltkrieg ging im Jahre 1918 zu Ende, und mit ihm auch die Habsburgermonarchie. Führende tschechische Politiker suchten Wege zur Machtübernahme in einem stark autonomen oder sogar selbstständigen tschechischen bzw. tschechoslowakischen Staat. Im Juli 1918 wurde der Nationalausschuss [Národní výbor] gegründet, dessen 38 Sitze nach den Reichsratswahlergebnissen von 1911 unter den Parteien verteilt

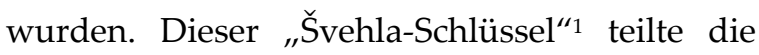

\footnotetext{
* Schriftfassung eines Vortrages, den der Verfasser am 19. 11. 2008 auf der vom Institut für Rechts- und Verfassungsgeschichte der Rechtswissenschaftlichen Fakultät der Universität Wien und vom Österreichischen Staatsarchiv veranstalteten Tagung "Staatsgründung und Verfassungsordnung“ in Wien gehalten hat. Der Verfasser und die Herausgeber danken dem Institutsvorstand, Prof. Thomas Simon, für seine
} 
Mandate den einzelnen Parteien zu; diese entschieden dann, wer die Mandate bekommen sollte, ohne darauf Rücksicht zu nehmen, wer dem Reichsrat persönlich angehörte. Der „Švehla-Schlüssel“" wurde im November 1918 im Rahmen der provisorischen Verfassung (Gesetz Nr. 37/1918 Sb. vom 13. November 1918) noch einmal bei der Erweiterung des Nationalausschusses angewendet; so entstand die Nationalversammlung mit 256 Abgeordneten. Wir werden sehen, wie sich der Gedanke, dass über die Mandate die Parteien zu verfügen hätten, auch später behauptete.

Am 28. Oktober 1918 verbreitete sich in Prag die Nachricht, dass die letzte österreichischungarische Regierung die Friedensbedingungen des US-Präsidenten Wilson akzeptiert hatte. Dies wurde als Kapitulation der Monarchie verstanden. Der Nationalausschuss und sein Präsidium reagierten schnell mit der Übernahme der Macht und proklamierten den unabhängigen Staat. Im sog. Rezeptionsgesetz (Gesetz Nr. 11/1918 Sb. vom 28. Oktober 1918) wurde die gesamte staatliche Verwaltung dem Nationalausschuss unterstellt und die rechtliche Kontinuität gesichert; die Frage der Staatsform blieb jedoch offen. In den folgenden Tagen legte der Nationalausschuss in einer Serie von Gesetzen die Grundlagen des neuen Staates. Am letzten Tag seiner Existenz, dem 13. November 1918, verabschiedete er die provisorische Verfassung (Gesetz Nr. 37/1918 Sb.). Am nächsten Tag, dem 14. November, trat die Nationalversammlung zum ersten Mal zusammen, setzte die Habsburger vom böhmischen Thron ab, proklamierte die Republik und wählte ihren Präsidenten sowie die Regierung.

Zustimmung zur Veröffentlichung des Beitrages in dieser Zeitschrift.

${ }^{1}$ Benannt nach A. Švehla, dem führenden Politiker der Agrarpartei, Vizepräsidenten des Nationalausschusses und späteren tschechoslowakischen Ministerpräsidenten.
Wie sah das System der provisorischen Verfassung aus? Das sehr knappe Gesetz über die provisorische Verfassung beinhaltet in 21 Paragraphen Bestimmungen über die Nationalverfassung, den Präsidenten der Republik und die Regierung. Dieses Provisorium galt bis Ende Februar 1920, als die definitive Verfassung vom 29. Februar 1920 verabschiedet wurde. Erst danach, im Mai 1920, fanden die ersten Parlamentswahlen statt. Das System der provisorischen Verfassung und seine Änderungen im Jahre 1919 beeinflussten die weitere Verfassungsentwicklung.

Vor allem wurde, wie bereits gesagt, der sog. „Švehla-Schlüssel“ wiederholt: „§ 1: Der Nationalausschuss verbreitert sich auf 256 Mitglieder nach der Art und Weise und dem Schlüssel, die für den Nationalausschuss angewendet wurden. Diese Körperschaft nennt sich die Nationalversammlung. §2: Dieselbe Art und Weise und derselbe Schlüssel gelten auch für eine Ergänzung der Nationalversammlung, falls irgendein Mitglied entfällt", beginnt die provisorische Verfassung. Im April 1919 verließen zwei sozialdemokratische Abgeordnete, Modráček und Hudec, ihre Partei. Die Partei berief beide Herren $\mathrm{ab}$ und benannte neue Abgeordnete; der Verfassungsausschuss der Nationalversammlung hat dieses Vorgehen am 21. Mai bestätigt. ${ }^{2}$ So wurde auch praktisch gezeigt, dass Mandate, mindestens in der Revolutionären Nationalversammlung, den Parteien gehörten. In veränderter Form hat diesen Grundsatz auch das definitive Verfassungssystem übernommen.

Die provisorische Verfassung begründete eine Versammlungsregierung, also eine Regierungsform, in der die Regierung vom Parlament gewählt und von ihm völlig abhängig ist. Wahrscheinlich wollte sich das revolutionäre Parla-

\footnotetext{
${ }^{2}$ Rede des Abg. Franke, Sten.Prot., 56. Sitzung, 28. 5. 1919.
} 
ment die Macht mit niemandem teilen, ${ }^{3}$ aber es gab auch praktische Gründe für dieses Vorgehen: Der neu gewählte Präsident der Republik, T. G. Masaryk, blieb zunächst im Ausland (er kam erst am 21. Dezember 1918 nach Prag) und hätte Schwierigkeiten gehabt, eine Regierung zu ernennen. So argumentierte auch der faktische Autor der provisorischen Verfassung, der sozialdemokratische Abgeordnete A. Meissner, in einer Debatte vom Mai 1919. ${ }^{4}$

Erst nach seiner Ankunft hat T. G. Masaryk eine Verfassungsänderung durchgesetzt, die das klassische Modell einer parlamentarischen Republik widerspiegelte: Das Gesetz Nr. 271/1919 Sb. vom 23. Mai 1919 gab dem Präsidenten die dafür typischen Befugnisse, den Ministerpräsidenten und die Minister zu ernennen und zu entlassen ( $\$ 14$ der provisorischen Verfassung, neue Fassung); außerdem konnte die Nationalversammlung durch ein Misstrauensvotum nur die gesamte Regierung stürzen, nicht aber einzelne ihrer Mitglieder ( $\$ 16$ der provisorischen Verfassung, neue Fassung). Diese Verfassungsänderung präzisierte und verbesserte auch in anderer Hinsicht die Position des Präsidenten.

\section{Vorbereitung der definitiven Verfassung}

Die Nationalversammlung verabschiedete die definitive Verfassung erst am 29. Februar 1920; dringliche Probleme der Konsolidierung der Verhältnisse im neuen Staat wurden bevorzugt behandelt. Den Verfassungsentwurf bereitete das Innenministerium in enger Zusammenarbeit mit dem Verfassungsausschuss der Nationalversammlung seit Herbst 1919 vor. Die wichtigsten und gut dokumentierten Debatten fanden im

\footnotetext{
${ }^{3}$ So MALÝ u.a., Dějiny 273, sowie KLIMEK, Velké dějiny 13,53 .

${ }^{4}$ Rede des Abg. Meissner, Sten.Prot., 54. Sitzung, 23. 5. 1919.
}

Januar und Februar 1920 im Verfassungsausschuss statt. Die führenden Persönlichkeiten bei der Vorbereitung der Verfassung waren der Innenminister A. Švehla, der Sektionschef im Innenministerium J. Hoetzel, Professor des Verfassungsrechts und Hauptautor des Verfassungstextes, außerdem der Vorsitzende des Verfassungsausschusses A. Meissner, Autor der provisorischen Verfassung, sein Vize, Professor F. Weyr, und der Berichterstatter des Verfassungsausschusses, V. Bouček. ${ }^{5}$

Die Verfassung, genauer: die Verfassungscharta der Tschechoslowakischen Republik (Gesetz Nr. 121/1920 Sb. vom 29. Februar 1920) konstituierte das klassische System einer parlamentarischen Republik. Die Nationalversammlung hatte zwei Kammern, das Abgeordnetenhaus und den Senat. Beide wurden in ziemlich langen Wahlperioden (sechs bzw. acht Jahre) nach dem direkten und gleichen Wahlrecht für alle Männer und Frauen gewählt (§ 6-17); der Senat blieb jedoch ohne jede faktische Bedeutung. Die Regierung, ernannt und entlassen vom Präsidenten der Republik (§70), war dem Abgeordnetenhaus verantwortlich; es gab die Möglichkeit eines Misstrauensvotums (§ 78). Der Präsident wurde von der Nationalversammlung alle sieben Jahre gewählt, niemand (ausgenommen der erste Präsident, also T.G. Masaryk) durfte mehr als zweimal gewählt werden (§56-58). Alle Befugnisse des Präsidenten waren jedoch durch eine Gegenzeichnungspflicht mit der Verantwortlichkeit der Regierung verbunden (§68). Der Präsident der Republik konnte beide Kammern des Parlaments auflösen (§31). Falls die Kammern nicht tagten, übernahm in dringlichen

\footnotetext{
${ }^{5} \mathrm{Zu}$ den Diskussionen im Verfassungsausschuss siehe BROKLOvÁ, První ústava, sowie DiEs., Prezident. Vgl. auch die Begründung des Verfassungsentwurfes im Bericht des Verfassungsausschusses zur Verfassungscharta der Tschechoslowakischen Republik, Drucksache 2421, und in der Rede des Abg. Bouček, Sten.Prot., 125. Sitzung, 27. 2. 1920.
} 
Fällen ein Ständiger Ausschuss die Ersatzgesetzgebung. Im Ständigen Ausschuss saßen 16 Abgeordnete und acht Senatoren; seine Maßnahmen standen unter der obligatorischen Kontrolle des Verfassungsgerichts (§ 54). Die Regierung konnte bei einem eigenen Gesetzesentwurf die Ablehnung des Parlaments mit Hilfe eines Referendums überwinden (§46); ein solches Referendum fand jedoch nie statt.

Am selben Tag, dem 29. Februar 1920, wurden auch weitere wichtige Gesetze erlassen, die die Gestalt des tschechoslowakischen Parlamentarismus mitbestimmten: die Wahlordnung (Gesetz Nr. 123/1920 Sb.), das Gesetz Nr. 124/1920 $\mathrm{Sb}$. über die Zusammensetzung und Befugnisse des Senats, und das Gesetz Nr. 125/1920 Sb. über das Wahlgericht.

Der wichtigste Unterschied zur alten österreichischen Verfassung lag in einer massiven Stärkung der Legislative gegenüber der Exekutive. Es ging vor allem um die politische Verantwortung der Regierung gegenüber dem Abgeordnetenhaus, das die Regierung mit einem Misstrauensvotum stürzen konnte ( $\$ 78)$. Dieses Merkmal unterscheidet die neue parlamentarische Republik scharf von der alten konstitutionellen Monarchie. Das Parlament wurde auch vor einer etwaigen Nichteinberufung geschützt: $\operatorname{Im} \S 28$ wurde ausdrücklich geregelt, dass der Präsident der Republik zweimal im Jahre beide Kammern des Parlaments einberufen müsse, und zwar im März und im Oktober. Einen negativen Bezug auf die österreichischen Verhältnisse beinhaltete die Begründung des $§ 20$, der den Abgeordneten erst ein Jahr nach Beendung ihrer Tätigkeit als Parlamentarier erlaubt, eine Stelle im Staatsdienst anzutreten, „damit Mitglieder der Nationalversammlung nicht, wie es in Österreich der Fall war, ihr Mandat zugunsten einer ungerechtfertigten Karriere missbrauchen könnten. ${ }^{\text {"6 }}$ In

\footnotetext{
${ }^{6}$ Bericht des Verfassungsausschusses zur Verfassungscharta der Tschechoslowakischen Republik, Drucksache 2421.
}

den Zusammenhang der Stärkung der Legislative gehört auch die Neuregelung der Notgesetzgebung (siehe unten). Weitere Unterschiede finden wir in der Demokratisierung des Verfassungsrechtes, vor allem in der Verwirklichung des allgemeinen und gleichen Wahlrechts für Männer sowie Frauen.

Im Folgenden werden einige wichtige Themen vorgestellt, die im Verfassungsausschuss und während der Vorbereitungsarbeiten heftig diskutiert worden sind. Mit dem Parlamentarismus sind eine Reihe weiterer Fragen verbunden: nach der Existenz des Senats, nach der Rolle des Präsidenten, nach dem Ständigen Ausschuss und nach dem Wahlgericht. Die Verhältnisse in der Habsburgermonarchie dienten in den Diskussionen um diese Fragen oft als negatives Vorbild.

Es war seit langem umstritten, ob der neue Staat eine zweite Parlamentskammer, den Senat, brauche. Der Verfassungsausschuss hat sich nach langen und schwierigen Diskussionen für ein Zweikammersystem ausgesprochen; bestimmend dabei war die Angst vor einer Allmächtigkeit und möglichen Fehlentscheidungen des Unterhauses. Die Sozialdemokratie war dagegen, die rechten Parteien waren dafür, die Nationaldemokraten sprachen sich sogar für eine Kombination aus Wahl und Ernennung der Senatoren durch den Präsidenten aus. Der Kompromiss lag darin, dass der Senat genauso demokratisch legitimiert sein musste wie das Abgeordnetenhaus, also - im Gegensatz zum Wiener Herrenhaus - aus allgemeinen, direkten und gleichen Wahlen hervorgehen musste. Gerade die Bemühungen, Parallelen zum Herrenhaus zu vermeiden, führten zu einer schädlichen Ähnlichkeit beider Kammern. Für den Senat war ein höheres Mindestalter für das aktive (26 Jahre) und passive (45 Jahre) Wahlrecht vorgesehen, zweimal weniger Mitglieder (150) und eine längere Wahlperiode (acht Jahre). Das reichte nicht aus; der Senat blieb eine bloße Kopie des 
Abgeordnetenhauses ohne jede faktische Bedeutung. ${ }^{7}$

Auch die Position des Präsidenten war Gegenstand von Streitigkeiten. Einerseits sprach die enorme Autorität von T. G. Masaryk dafür, dem Präsidenten ziemlich breite Befugnisse einzuräumen. Andererseits wollte der Verfassungsausschuss eine „kaiserliche“ Stellung des Präsidenten vermeiden: „Es wurde bei uns nach einem starken Präsidenten nach amerikanischem Vorbild gerufen, und die enge Zusammenarbeit Masaryks mit Wilson wies in diese Richtung, aber der Verfassungsausschuss widerstand dieser Versuchung, im Gedanken an die Zukunft und gewarnt von den Gesetzwidrigkeiten, von denen die Regierung Kaiser Franz Josephs geradezu strotzte. ${ }^{\text {} 8}$ Der Präsident hatte zwar starke Befugnisse, er konnte zum Beispiel jederzeit das Abgeordnetenhaus und den Senat auflösen, jedoch waren alle seine Akte gegenzeichnungspflichtig und damit an die Verantwortlichkeit der Regierung gekoppelt ( $\$ \S 66$ und 68).

Falls die Kammern des Parlaments nicht tagten, übernahm, wie schon gesagt, der Ständige Ausschuss die Ersatzgesetzgebung in dringlichen Fällen. Im Ständigen Ausschuss saßen 16 Abgeordnete und acht Senatoren. Die Maßnahmen des Ständigen Ausschusses standen unter der obligatorischen Kontrolle des Verfassungsgerichts; der Präsident hatte ihnen gegenüber ein absolutes Vetorecht ( $\$ 54)$. Die Argumentation für diese Lösung wendete sich gegen die kaiserlichen Notverordnungen nach dem berühmten $\S 14$ der Dezemberverfassung von 1867.9 Die Notgesetzgebung in der Tschechoslowakei ging von der Exekutive auf ein Organ des Parlaments

\footnotetext{
${ }^{7}$ BROKLOVÁ, První ústava 85ff. Ausführlich zu diesen Debatten KYSELA, Dvoukomorové systémy 363ff.

${ }^{8}$ Bericht des Verfassungsausschusses zur Verfassungscharta der Tschechoslowakischen Republik, Drucksache 2421.

${ }_{9}^{9}$ Rede des Abg. Bouček, Sten.Prot., 125. Sitzung, 27. 2. 1920.
}

über, eine Kontrolle der Verfassungsmäßigkeit wurde installiert und es wurde unzweideutig geregelt, dass eine Maßnahme ihre Gültigkeit verlor, falls ihr beide Kammern des Parlaments nicht innerhalb von zwei Monaten nach Beginn der Sitzungen zustimmten (§ 54 Abs. 15).

Sehr umstritten war der $\S 13$ des Gesetzes über das Wahlgericht, wonach das Wahlgericht einem Abgeordneten das Mandat entziehen durfte, wenn er „aus niedrigen und unfairen Gründen" seine Parteizugehörigkeit verloren hatte. Das Wahlgericht arbeitete unter dem Vorsitz des Präsidenten des Obersten Verwaltungsgerichts, die anderen Richter wählte das Abgeordnetenhaus. In der Praxis hat das Wahlgericht dann tatsächlich in mehreren Fällen einen Mandatsverlust ausgesprochen. Diese Regelung fand breite Zustimmung bei den Parteien. Das Wahlgericht wich zwar von der bisherigen Praxis der provisorischen Verfassung mit ihrem automatischen Mandatsverlust ab, hielt aber an der Möglichkeit dazu fest: „Dies entspricht dem Verhältniswahlgrundsatz, denn falls ein Abgeordneter aus seiner Wahlpartei austritt, verliert die Wählerschaft dieser Partei ihre Vertretung. ${ }^{\prime 10}$ Es war für den Verfassungsausschuss aber nicht denkbar, jeden Parteiaustritt mit Mandatsverlust zu bestrafen, denn manchmal kann ein solcher Austritt dem Willen der Wählerschaft oder eines Teiles davon entsprechen. Deswegen sollte das Wahlgericht die Gründe des Austritts berücksichtigen.

Der Verfassungsentwurf wurde von 27. bis 29. Februar 1920 im Plenum der Nationalversammlung beraten; in der Diskussion ging es vor allem um die Macht der Parteien im Verfassungssystem. Der Chef der Nationaldemokraten und bis Juli 1919 Ministerpräsident, K. Kramáŕ, sprach sogar von einer „Verfassung der Parteiautokratie“. Er kritisierte vor allem das neue

${ }^{10}$ Bericht des Verfassungsausschusses zum Regierungsentwurf des Gesetzes über das Wahlgericht, Drucksache 2424. 
Wahlgesetz, das die streng gebundenen Wahllisten einführte, die den Wählern keine Möglichkeiten gaben, über einzelne Kandidaten zu entscheiden. ${ }^{11}$

Kramář behauptete zutreffend, dass die Verfassung und andere Gesetze mit der Verhältniswahl, dem Wahlgericht und den streng gebundenen Wahllisten die Macht der Parteien in der Tschechoslowakei verstärkten. Dazu trugen auch faktische Umstände bei - von 1920 bis 1925 die Notwendigkeit, eine breite Koalition aller fünf tschechoslowakischen demokratischen Parteien zu bilden (die sogenannte „Pětka“), danach Koalitionen mehrerer tschechoslowakischer und deutscher Parteien, die auch zu einer extrem schwierigen Kompromisssuche führten.

Kramář kritisierte den Verfassungsentwurf vom rechten Flügel der politischen Szene her. Vom anderen Flügel her kritisierten manche linke Sozialdemokraten die (ziemlich schwache, siehe oben) Position des Präsidenten in der Verfassung als "monarchistisch"; der Abgeordnete E. Burian sprach in der Verfassungsdebatte von einer monarchistischen Verfassung ohne Monarchen: „Wir schreiben sehr viel von der alten Habsburgermonarchie und ihren Regeln ab. [...] Ein Beispiel: die Rechte, die wir dem Präsidenten geben. Wir geben ihm ähnliche Rechte, wie sie im alten Österreich der Herrscher hatte: das Recht, das Parlament aufzulösen, das Recht, die Regierung zu ernennen, das Recht, Minister zu ernennen. [...] Bei uns wird sich unser Abgeordnetenhaus Einfluss und Macht mit dem Präsidenten, mit der Regierung, mit dem Senat teilen müssen; bei uns ist immer das Motto: ein starker Präsident, eine starke Regierung, ein starker Senat usw. zu hören. Das ist mit der Idee der Volkssouveränität kaum vereinbar." ${ }^{12}$ Es ist verständlich, dass linke Sozialdemokraten wie

\footnotetext{
${ }^{11}$ Rede des Abg. Kramář, Sten.Prot., 125. Sitzung, 27. 2. 1920

12 Rede des Abg. Burian, Sten.Prot., 126. Sitzung, 28. 2. 1920.
}

E. Burian eher das System der Versammlungsregierung als die klassische parlamentarische Republik wollten. Die Mehrheit der damaligen Politiker sprach sich jedoch für den klassischen Grundsatz der Gewaltenteilung aus, der dem Verfassungssystem, das sie in der Habsburgermonarchie so gut kennenlernen konnten, viel näher war.

\section{4. Ähnlichkeiten und Unterschiede des tschechoslowakischen Parlamentarismus im Vergleich mit der Habsburgermonarchie}

Die parlamentarische Tradition in Böhmen und Mähren begann im Jahre 1848 mit dem verfassungsgebenden Reichstag von Kremsier [Kroměř́̌ž]; seit 1861 ist die Tradition fast ununterbrochen. Führende Politiker Böhmens und Mährens dienten als Abgeordnete im Wiener Reichsrat oder im böhmischen oder mährischen Landtag. Die meisten Gründer der Republik von 1918 hatten parlamentarische Erfahrung; so war z.B. der erste tschechoslowakische Ministerpräsident, K. Kramář, im turbulenten Jahr 1897 Vizepräsident des Abgeordnetenhauses des Reichsrates.

Wie allgemein bekannt, litt der österreichische Parlamentarismus (sowohl im Wiener Abgeordnetenhaus, als auch im böhmischen Landtag) seit den neunziger Jahren des 19. Jahrhunderts unter gravierenden Obstruktionen. Der Reichsrat reagierte mit der sog. Lex Falkenhayn von 1897, der sog. Lex Kramár von $1909^{13}$ und mit einer neuen Geschäftsordnung (Gesetz vom 11. Juni 1917, betreffend die Geschäftsordnung des Reichsrates, RGB1. 253/1917).

\footnotetext{
${ }^{13}$ BERNATZIK, Verfassungsgesetze 840-844.
} 
Es ist interessant zu sehen, welche Regeln des österreichischen Parlamentarismus die neue Republik übernahm und welche nicht. Verglichen werden im Folgenden einerseits das Gesetz vom 12. Mai 1873 (RGBl. 94/1873) betreffend die GO des Reichsrates sowie die GO für das Abgeordnetenhaus des Reichrates vom 2. März 1875, andererseits die GO des Abgeordnetenhauses der Nationalversammlung (Gesetz vom 15. April 1920, Nr. 325/1920 Sb.).

Die Nationalversammlung übernahm vom Reichsrat viele praktische Regelungen, die auch heute nützlich wären. So war das Recht der Abgeordneten, selbstständig Anträge zu stellen, dahingehend beschränkt, dass die Unterstützung von mindestens 20 weiteren Abgeordneten vorliegen musste (§ 18 GO 1875 und §§ 19, 48 GO 1920). Diese Regelung schützte das Parlament vor einer Welle von aussichtslosen Anträgen. $\mathrm{Zu}$ einer größeren Effizienz der parlamentarischen Arbeit führte auch die Möglichkeit, bereits vor der Erschöpfung der Rednerliste eine Debatte zu beschließen (nach zwei Reden im $\S 39$ GO 1875, nach vier Reden im $\S 48$ Abs. 2 GO 1920); in beiden Fällen mit noch einer Rede für und gegen den Antrag. Auch die abwechselnde Erteilung des Wortes an Redner "für" und Redner "gegen" einen Antrag verbindet die österreichische und die tschechoslowakische Parlamentsarbeit (§ 52 GO 1875, § 44 GO 1920). Die neutrale Stellung des Präsidenten des Hauses, des „unparteiischen Beschützers des Parlamentslebens ${ }^{\text {"14 }}$ wird dadurch unterstrichen, dass der Präsident sich nicht an der Abstimmung beteiligt ( 84 GO 1875, §56 Abs.7 GO 1920). Alle diese Regelungen, die dem heutigen tschechischen Parlamentsrecht fremd sind, trugen zu einer guten parlamentarischen Kultur bei. ${ }^{15}$

Die Tschechoslowakei übernahm auch die Lex Falkenhayn, die dem Präsidenten ermöglichte,

\footnotetext{
${ }^{14}$ Bericht des Verfassungsausschusses zur Änderung der GO, Nationalversammlung, Drucksache 2750.

${ }^{15}$ WINTR, Parlamentní kultura.
}

grob störende Abgeordnete für drei Sitzungen bzw. mit Zustimmung des Hauses, für dreißig Tage aus dem Hause auszuschließen. In der Nationalversammlung durfte ein Abgeordneter bis zum Ende der Sitzung und, falls er die weiteren Verhandlungen unmöglich machte, für höchstens zehn Sitzungen ausgeschlossen werden (§ 52 GO 1920). Die Wiener Lex Falkenhayn wurde bald zurückgenommen ${ }^{16}$ und fehlte leider später angesichts vieler Störaktionen; das tschechoslowakische Parlament war gegen die kommunistische und deutschnationalistische Obstruktion besser gerüstet.

Während in den Diskussionen über die Verfassung Bezüge auf die Verhältnisse in der Monarchie eher selten waren, wurde bei der Vorbereitung der Geschäftsordnung oft das Wiener Parlament angesprochen. In der Gesetzesbegründung der neuen Geschäftsordnung wurde angedeutet, dass die österreichischen Regierungen das Parlament zugunsten der Dynastie hätten schwächen wollen und dass Parteienkonflikte im Parlament dazu als willkommenes Mittel gedient hätten. ${ }^{17}$ Daraus folgt für den Berichterstatter O. Krouský, dass die neue Geschäftsordnung viel effizienter Obstruktionen bekämpfen und die Parlamentsarbeit schützen müsse. ${ }^{18}$

Vor allem die Beratung von Gesetzesvorlagen und die Dringlichkeitsanträge wurden anders geregelt als in der Monarchie. Der Vorrang von Regierungsvorlagen vor anderen Gegenständen ( 5 des Gesetzes RGBl. 94/1873) galt in der Republik nicht mehr; eine solche Regelung wurde als undemokratisch verworfen. Einen Dringlichkeitsantrag konnte nicht jeder Abgeordnete stellen (§ 42 GO 1875), sondern nur die Regierung, der Präsident des Hauses oder zwei Fünftel seiner Mitglieder (§ 55 GO 1920); diese Bestim-

\footnotetext{
${ }^{16}$ BERNATZIK, Verfassungsgesetze 841.

17 Bericht des Verfassungsausschusses zur Änderung der GO, Nationalversammlung, Drucksache 2750.

${ }^{18}$ Rede des Abg. Krouský, Sten.Prot., 144. Sitzung, 14. 4. 1920.
} 
mung sollte Obstruktionen verhindern. Der Mandatsverlust aufgrund längerer unerlaubter Abwesenheit eines Abgeordneten ( $\$ 4$ des Gesetzes RGBl.94/1873) wurde ebenfalls abgeschafft.

Die Geschäftsordnung von 1920 regelte explizit die Frage der Fraktionen im Parlament. Die Fraktionsangehörigkeit spielte eine Rolle bei der Rednerreihenfolge (§ 45) und bei der Wahl der Ausschüsse, die dem Grundsatz der Verhältniswahl folgte (§ 63). Die älteren Vorschriften kannten dagegen keine Fraktionen.

Wie man sieht, blieben die traditionellen parlamentarischen Regeln im Wesentlichen unverändert. Erst die totalitären Regime brachen diese Tradition.

\section{Schluss}

Die tschechoslowakische Verfassungsentwicklung nach dem Ersten Weltkrieg kann nicht ohne Bezug auf das Verfassungsmodell der Habsburgermonarchie verstanden werden. Obwohl sich der neue Staat in vieler Hinsicht scharf vom "alten Regime“ abgrenzen wollte, blieb doch Vieles von der österreichischen Tradition. Neben den parlamentarischen Regeln, die bereits angesprochen wurden, galt dies auch für weitere Teile der Verfassung; so übernahm die Verfassung vom 29. Februar 1920 viele Bürgerrechte der Dezemberverfassung von 1867 und ausdrücklich auch ihre Regelung der Gerichtsbarkeit: „[...] Vorschriften des österreichischen Gesetzes von 1867 gehören nach Inhalt sowie Form zu den besten Kodifikationen dieser Art. Weil die Staatsumwälzung die Gerichtsbarkeit viel weniger berührt als die Staatsverwaltung, war für die Verfassungsvorschriften über die Gerichtsbarkeit möglich, das angesprochene österreichische Gesetz im Vielem zur Grundlage zu nehmen. ${ }^{\prime 19}$

Unterschiede zwischen dem alten österreichischen und dem neuen tschechoslowakischen Verfassungssystem müssen also nicht als Ausdruck eines Zerstörungswillens der Revolutionäre interpretiert werden. Vielmehr ging es, ebenso wie im gesamten damaligen Europa, um eine natürliche Weiterentwicklung der Verfassungsformen. Aus heutiger Sicht verstehen wir beide Verfassungssysteme eher als Ausprägungen derselben Tradition, zwei Glieder einer Kette, die nur die Zeit voneinander unterscheidet.

\section{Korrespondenz:}

JUDr. PhDr. Jan Wintr, Ph.D.

Univerzita Karlova v Praze

Právnicka Fakulta

nám. Curieových 7, 11640 Praha 1, Česká republika wintr@prf.cuni.cz

\footnotetext{
${ }^{19}$ Bericht des Verfassungsausschusses zur Verfassungscharta der Tschechoslowakischen Republik, Drucksache 2421.
} 


\section{Abkürzungen:}

GO

Geschäftsordnung

$\mathrm{Sb}$ Gesetzsammlung der

Tschechoslowakei

Sten.Prot. Stenographische Protokolle

\section{Literatur:}

Edmund BERNATZIK, Die österreichischen Verfassungsgesetze (Wien ${ }^{2} 1911$ ).

Eva BroKlová (Hg.), První československá ústava. Diskuse v ústavním výboru v lednu a únoru 1920 (Praha 1992).
Eva BroKlová (Hg.), Prezident Republiky československé (Praha 2001).

Antonín KLIMEK, Velké dějiny zemí Koruny české, Bd. 13: 1918-1929 (Praha 2000).

Jan KYSELA, Dvoukomorové systémy (Praha 2004).

Karel MALÝ u.a., Dějiny českého a československého práva do roku 1945 (Praha 1997).

Jan WINTR, Česká parlamentní kultura (Praha 2010).

Die Berichte des Verfassungsausschusses zur Verfassungscharta der Tschechoslowakischen Republik sowie die Stenographischen Protokolle der Nationalversammlung sind in der gemeinsamen tschechisch-slowakische digitalen Parlamentsbibliothek zugänglich: [http://www.psp.cz] (abgerufen am: 22. 5. 2012). 